"Un breve recorrido por la bibliografía argentina en los estudios sobre la radio" Agustín Espada

Question/Cuestión, Vol 2 N66, Agosto 2020.

ISSN: 1669-6581

https://perio.unlp.edu.ar/ojs/index.php/question/

IICom - FPyCS - UNLP

\section{“UN BREVE RECORRIDO POR LA BIBLIOGRAFÍA ARGENTINA EN LOS ESTUDIOS SOBRE LA RADIO"}

\section{"A BRIEF TOUR OF THE ARGENTINE BIBLIOGRAPHY IN RADIO STUDIES"}

\section{Agustín Espada}

\section{Resumen}

Recorrido por materiales bibliográficos sobre la radio en Argentina, principales autores y exponentes. El surgimiento de la radio desde la perspectiva empresarial, estatal y comunitaria. La radio en los tiempos de la masificación de internet como plataforma de producción y circulación cultural.

\section{Palabras Clave}

Radio, referencias bibliográficas, comunicación comunitaria y universitaria, convergencia digital 


\section{Abstract}

Tour of bibliographic materials on radio in Argentina, main authors and exponents. The emergence of radio from the business, state and community perspective. Radio in times of the massification of the internet as a platform for cultural production and circulation.

\section{Keywords}

Radio, bibliographic references, community and university communication, digital convergence

\section{Introducción}

La radio ha tenido un lugar trascendente en la configuración de la comunicación como un campo de estudio propio dentro de las Ciencias Sociales. Tironeada y heredera de la sociología, la psicología, la historia, la semiótica y la lingüística, la comunicación ha logrado transformarse e instalarse como un campo disciplinario independiente pero complejo, heterogéneo y transdisciplinar. En esa línea, "la radio" como objeto de estudio dentro de ese campo ha realizado aportes más que importantes para la definición de las especificidades de estos trabajos.

Este breve artículo tiene el objetivo de hacer un racconto por algunas de los principales aportes académicos y las más destacadas formas de problematizar, abordar e investigar a la radio. Es un recorrido que no pretende ser exhaustivo y reconoce los límites y las falencias de mencionar autores y trabajos ante la posibilidad de omitir otros. Aclaración entonces: es un primer paso para reconstruir la historia de los estudios de la radiofonía en Argentina. Uno que se 
propone recolectar los trabajos realizados para contar la historia de las emisoras, problematizar las políticas públicas necesarias para el sistema, describir experiencias de radios comunitarias y universitarias, indagar en la incidencia cultural y social de la radio como vehículo de comunicación y, por último, la transformación digital y convergente del medio en internet.

Este artículo, entonces, se plantea los siguientes interrogantes: ¿Cuáles han sido las líneas de estudio más desarrolladas en el campo de la investigación sobre la radio en Argentina? ¿Cuáles son las problemáticas propias de este campo en el país? ¿Cómo se han trabajado y qué aportes se han realizado?

\section{Breve aclaración metodológica}

Este trabajo realiza un recorrido por la bibliografía que tiene a la radio, en sus distintas variantes, como objeto de estudio. No abarca la totalidad del campo ni pretende hacerlo. El trabajo de búsqueda y rastreo de trabajos debe continuar y ser alimentado a través del tiempo para destacar la relevancia de este objeto de estudio dentro del campo de la comunicación como ciencia social. La forma de acceder a estos trabajos fue internet y los distintos buscadores de artículos académicos así como también capítulos de libros u obras completas. Este artículo no cuenta con un recorte temporal ni geográfico aunque reconoce dos tipos de sesgos: presenta trabajos particularmente actuales y mayoritariamente elaborados desde Buenos Aires. Es un desafío a futuro construir un panorama mucho más amplio y completo de los estudios de radio en Argentina. 


\section{La relevancia sociocultural}

Para pensar la relevancia de la radio en el campo de los estudios de comunicación en Argentina hay que hablar, en primer lugar, de una referente ineludible: María Cristina Mata. Su trabajo, desde la provincia de Córdoba, y aporte desde distintos artículos de la Revista Signo y Pensamiento de la Universidad Javeriana de Colombia son piedras fundamentales para construir un estudio serio e integral sobre la radiofonía argentina. Las definiciones que Mata brindó sobre la radio son numerosísimas. Entre ellas se encuentran las siguientes: "en la radio se juega, al igual que en otros medios masivos, la constitución del espacio público, ese espacio hecho de normas y acuerdos que fundan la socialidad" (Mata, 1998); "en Argentina, donde mientras los gobernantes (...) exhiben como si fuese un mérito y no una obligación su respeto por la libre expresión, miles de emisoras de frecuencia modulada entre ellas muchas populares y comunitarias, existen al margen de la ley porque ni a los dueños del mercado comunicacional ni a los partidos políticos mayoritarios les interesa efectivamente liberar las ondas del modelo restrictivo" (Mata, 1994) para dar una fotografía absoluta de la realidad de la comunicación comunitaria en el país; o la distinción entre comunitario y popular para definir a ese sector de la comunicación "reconocerse populares implica un posicionamiento global frente a un sistema económico-social en el cual dichos sectores -sin importar de qué grupos se trate (...)- son marginados o excluidos también globalmente del poder" (Mata, 1993a),

No es fácil conciliar profesionalidad con protagonismo popular. No es fácil resistir la lógica del mercado -la de la máxima rentabilidadcuando al mismo tiempo se quiere valorar las expresiones culturales no comerciales. No es fácil asumir el debate, la confrontación de ideas como estrategia de comunicación democrática cuando no se 
han superado dogmatismos o cuando el sensacionalismo y la superficialidad informativa parecen estar ganando la batalla. (Mata, 1993b)

Pero los aportes en esta línea de trabajo tienen otros autores. Oscar Bosetti, por caso, escribió "Radiofonías" (1994) en un libro que relata la inserción social y transformación cultural del medio en sus inicios. Por el mismo camino se encuentra el trabajo de Eduardo Romano (1993), el de Ricardo Gallo (1991) o el trabajo de Ulanovsky y Merkin (1999; 2006) donde se destacan las principales figuras, programas y programaciones de la historia del medio.

Entre los trabajos de corte más académico, Ricardo Haye es un gran artífice de la relevancia cultural de la radio en la sociedad argentina (1998; 2000; 2003). Matallana (2006) escribió sobre el nacimiento del medio y su camino hasta transformarse en referente artístico, político y social en Argentina.

José Luis Fernández es de los autores e investigadores más prolíficos, profundos e innovadores en el campo de la comunicación radiofónica. Su trabajos sobre las particularidades del lenguaje radiofónico (1994; 2003), la evolución institucional del medio (2008), las particularidades de la escucha (2012) además de la coordinación de un gran espacio para pensar, entre otras cosas, lo radiofónico como la revista Letra, Imagen y Sonido (LIS). Pero además, Fernández realiza un gran aporte a las discusiones más actuales de la radio sobre su inserción en la convergencia en distintos trabajos sobre el networking (2016), los límites de lo audiovisual (2013) e incluso el podcasting (2017, 2018).

Fernández propone pensar en "lo radiofónico" a partir de la definición de "medio" como "todo dispositivo técnico o conjunto de ellos que -con sus 
prácticas sociales vinculadas- permiten la relación discursiva entre individuos y/o sectores sociales más allá del cara-a-cara" (2008:37). Así, va a entender luego lo radiofónico como un conjunto o serie de fenómenos: dispositivos técnicos, contenidos y prácticas sociales asociadas.

Dispositivos técnicos que se van a ir incorporando al uso radiofónico generando posibilidades y restricciones de construcción discursiva, géneros y estilos radiofónicos que van a ir apareciendo y consolidándose y lo mismo va a ocurrir con prácticas sociales nuevas -total o parcialmente, directa o indirectamente- relacionadas con el nuevo medio. (2008)

Es el mismo Fernández (2014) el que brinda tres definiciones organizadoras sobre los espacios que la radio puede generar como medios de comunicación. La primera es la "radio-transmisión" que se da cuando el medio reproduce un espacio social de existencia previa e independiente al fenómeno mediático. La segunda es la "radio-soporte" que se encuentra cuando no se percibe un espacio del otro lado del parlante o auricular sino sólo una voz individual o música. La tercera definición, y más pertinente con el medio radiofónico, es la de "radio-emisión". En este fenómeno, el espacio percibido a través del dispositivo sólo es accesible a través del medio. Este concepto de radio es definido como "una oferta discursiva en vivo, en interacción con lo grabado, generada por un centro emisor; con carga informativa variada, entrelazada con otros tipos discursivos, que se puede recibir realizando otras actividades que requieran visión y atención" (2014:98).

También existe una amplia bibliografía sobre las formas de producir radio. Desde los formatos informativos a los musicales, desde la producción a los lenguajes. Parte de estos trabajos pueden resumirse en los aportes de Portugal 
y Yudchak (2008) con una guía completa sobre roles productivos, equipos y formatos de radio. También puede encontrarse el trabajo de Atorresi (2005) sobre los géneros radiofónicos que incluye, también, un apartado práctico.

\section{Historia empresarial y políticas}

Hay una lista de libros muy interesantes sobre historia de diversas emisoras, principalmente de Buenos Aires. Con estos trabajos se pueden reconstruir distinta cuestiones: desde el entramado empresarial del mercado radiofónico a los criterios artísticos de programación, de la organización productiva a la relevancia sociocultural del medio en determinada época. La historia de la FM Rock \& Pop fue contada por Francisco Anselmi y Guillermo Courau (2015) en "La imaginación al poder"; "El Mundo, la radio", escrito por Martín Berrade (2009) relata el surgimiento y el auge de una de las emisoras más importantes de la historia de nuestro país; "Radio Belgrado" es el trabajo de Carlos Ulanovsky, Susana Pelayes, Alberto Ronzoni y Gustavo Lema (2014) sobre Radio Belgrano y su rol en la ebullición artística del retorno democrático en Argentina; "La radio Nacional, voces de la historia" es otro trabajo de Ulanovsky y Pelayes (2010) que cuenta la historia de la emisora del Estado Argentino; y esto se suma a otros tres libros escritos por Ulanovsky y equipo (1999, 2006 y 2007) sobre la historia artística y política de la radio en el país; Radio Rivadavia también tiene un libro propio editado en 1998 a propósito de los 40 años de la emisora; FM Metro también tiene un libro escrito por Pablo Wildau (2014) sobre los programas más representativos de la emisora; Paul Dougall (2005) escribió la historia familiar al frente de Radio Excelsior, otro faro del inicio y la época de oro de la radiofonía porteña. 
Otro gran trabajo, aunque no sobre una emisora, es del Andrea Matallana (2013) sobre la vida de Jaime Yankelevich y su importancia para el desarrollo cultural y comercial de la radio. En la misma línea se inscriben, por ejemplo, los trabajos de Marchi (2002) con entrevistas a personalidades de la radio porteña; "En mi vida" una autobiografía de Juan Alberto Badía (2012); un trabajo del mismo estilo sobre el conductor Fernando Bravo (2010); y también "Animal de radio" el trabajo de Lalo Mir y Carlos Barragán, por mencionar algunos.

Para pensar historias y evoluciones locales o regionales de sistemas radiofónicos pueden encontrarse trabajos de investigación sobre la ciudad de Rosario (Smerling, 2012), la provincia de Córdoba (Mata, 2000; Martínez Luque, 2011; 2012), San Juan (Olivares, 2018), San Luis (Toledo, 2018) Santa Fe (Levatti, 2018) de Jujuy (Brunet, 2003) o Salta (Picco, 2018).

En relación a las políticas de comunicación y su relación con la radiofonía se destaca el trabajo de compilación de Guillermo Mastrini (2009) en "Mucho ruido, pocas leyes" donde se cuenta, en sus primeros capítulos, el surgimiento del medio en nuestro país y los dubitativos pasos para su ordenamiento regulatorio. Además, Jorge Noguer (1985) también trabajó el marco normativo de la actividad así como también Horvath (1986) quien en "La trama secreta de la radiodifusión argentina" describe las discusiones y los problemas en torno al poder empresarial, político y la regulación. Este rápido panorama podría completarse con el trabajo de Elíades (2003), Tobi (2008) y Espada (2018) que comentan cómo se comportó la relación entre Estado y mercado en distintos momentos históricos.

Además, pueden ubicarse los trabajos del equipo interuniversitario comandado por María Soledad Seguro que analizan los impactos de la Ley de Servicios de Comunicación Audiovisual en los medios comunitarios (Segura, et.al., 2016, 
$2017,2018,2019)$. En el mismo rubro pueden insertarse los trabajos de Kejval $(2017,2019)$ e Iglesias $(2015 ; 2017)$.

\section{De las radios estatales a las comunitarias}

Las emisoras estatales en nuestro país han sido uno de los objetos de estudio más abordados por el campo de la comunicación radiofónica. Dentro de estos trabajos, los que se refieren a Radio Nacional, por lógica, son mayoría. Entre los más recientes se destacan los aportes de Linares para pensar el financiamiento, la gestión y las políticas de programación de las emisoras nacionales durante el kirchnerismo y el macrismo (Linares, 2017, 2020; Linares y Mallimaci, 2019). También se puede ubicar en este espacio los aportes de Monje, Mercadal y Doyle (2013) y la historia narrada por Avilés Rodilla (2016). Las radios universitarias han sido otro de los grandes temas de articulación e investigación del campo.

Las particularidades de las radios universitarias (Casajús, 2011 y 2018; y los trabajos coordinados por Bosetti y Haye en 2015, 2016 y 2017) y las particularidades de la comunicación comunitaria han sido muy trabajadas y abordadas por el campo de la comunicación en Argentina. En esa línea se destacan los aportes de Lamas y Villamayor (1998) con su manual sobre gestión de emisoras comunitarias, un trabajo completo y detallado sobre las dimensiones que deben considerarse al momento de conducir este tipo de proyectos. Otro trabajo ineludible es el de Kejval (2009) sobre el recorrido histórico, político y social de las radios comunitarias en Argentina y un título que hace referencia a su conceptualización: "Truchas".

Martín Iglesias -como Kejval (2017), Lamas (1996) y Villamayor (2014)combina y proviene de la hechura y la gestión de los medios comunitarios para 
insertarse en el campo académico desde el estudio y la transformación de la experiencia en objeto de estudio. En su libro y tesis "A contramano" (Iglesias, 2015) trabaja los modelos de gestión, organización e interacción con el Estado de diferentes radios comunitarias. Otros trabajos que pueden ser mencionadas en esta línea son los de Gerbaldo (2010) y Jaimes (2013; 2020).

Entre los trabajos más recientes se encuentra el de Binder, Fisher y Godinez Galay (2017) sobre las estrategias y procesos de generación de sostenibilidad económica y administrativa de radios comunitarias en el país. Este libro es un gran aporte por problematizar y trabajar cuestiones fundamentales de estos proyectos como los conceptos de "trabajo", "publicidad" y "organización".

Las radios universitarias son otro objeto de estudio habitual dentro del campo. Los trabajos de Lucía Casajús $(2011 ; 2014 ; 2015 ; 2016)$ pueden ser tomados como ejemplo de ello ya que no solo problematizan su razón de ser, su gestión y sus contenidos sino que también avanzan sobre sus estrategias para afrontar la convergencia digital. El trabajo de Ricardo Haye (2018) nuevamente se encuentra como imprescindible para comprender las particularidades de este sector de la radiofonía al igual que el de Aldo Rotman (2014) y Carlos Milito.

Por último, resulta indispensable para pensar tanto la comunicación universitaria, como la comunitaria y estatal en general los aportes y discusiones que tienen lugar en las Jornadas Universitarias de La Radio del Nuevo siglo organizadas anualmente por Oscar Bosetti y Ricardo Haye que cuentan con cuatro publicaciones diversas $(2015 ; 2016 ; 2018 ; 2020)$. 


\section{La radio en la convergencia digital}

Pensar la radio en los tiempos de la masificación de internet como plataforma de producción y circulación cultural trajo una gran cantidad de nuevas preguntas y formas de tomar al medio como objeto de estudio.

Ricardo Haye $(1999 ; 2003)$ fue uno de los primeros en presentar las discusiones, los desafíos y las oportunidades que habría el escenario digital para la radiofónica. Y también fue uno de los grandes presentadores locales de un objeto de estudio que hoy se encuentra más vigoroso que nunca: el podcasting.

La radio sincrónica, esa emisión de flujo continuo las 24 horas, los siete días de la semana, pierde exclusividad como objeto de estudio. Ahora debe compartir el lugar con la radio diacrónica que posibilita el podcasting.

Ya no es posible trabajar solamente sobre los grandes bloques horarios, que concentraban audiencias masivas ofreciéndoles productos efímeros. La comprensión acabada de la radio de nuestros días exige que también nos ocupemos de los productos discontinuos, de menor duración pero de vida más larga, producidos on demand para atender audiencias muy específicas y localizadas (Haye, 2011).

Los trabajos de Lucía Casajús $(2014 ; 2015)$ también deben ser tomados como referencia para el estudio de la adaptación a medios sociales principalmente de emisoras universitarias. Su trabajo junto a Carlos Milito (2009) realizó un aporte central para la definición de lo radiofónico en internet con una revisión bibliográfica sobre las conceptualizaciones. 
Para estudiar la radio en Internet de una forma completa y coherente, la investigación del fenómeno debe abordarse desde una mirada global e integradora dentro del modelo de comunicación multimedia combinándolo con el aporte de una mirada particular y específica en la materia. Así, al observar y analizar el fenómeno de la radio en Internet también deben estudiarse elementos tales como las características de Internet como plataforma (navegación, interactividad e hipertextualidad), las particularidades del lenguaje, la programación, la locución, la escritura, los diseños, los rasgos de los géneros periodísticos radiofónicos, los formatos para la organización de contenidos y las formas de acceso y consumo de esos productos informativos.

Creemos, que en este camino, se deben analizar situaciones concretas, más allá de luego ponerlas en contexto con una situación general de las distintas dimensiones del fenómeno en la búsqueda de la elaboración de un mapa que dé cuenta de los modelos actuales de radio en Internet, para realizar comparaciones y extraer diferenciaciones. La investigación de este fenómeno requiere un análisis exhaustivo de la dimensión de la comunicación interactiva, de su sistema expresivo sonoro y multimedia, de la incorporación de nuevas herramientas.

María José Müller es otra de las autores de trascendencia al momento de los estudios de la radiofonía en internet. Más allá de sus trabajos sobre los géneros y contenidos del prime-time radiofónico (Müller, 2016; 2018), ha realizado estudios muy importantes en relación a la adaptación de las emisoras musicales a internet (Müller, Martínez Costa y Corda, 2016; Müller y Martínez 
Costa, 2017) y también sobre las estrategias de podcasting en emisoras públicas (Müller, Martínez Costa y Villar, 2020).

En línea con los análisis y estudios de casos de adaptación de emisoras a los entornos digitales se destacan los trabajos de Avilés Rodilla sobre Radio Nacional (2015) y Radio Continental (2011), Vorterix (Espada, 2015), el de Linares también sobre Radio Nacional (2017), un trabajo sobre Radio Continental y la AM 750 (Amdán, et.al, 2014), un estudio sobre las estrategias en radios comunitarias como La Tribu y FM Boedo (Retegui y Amdán, 2017) y las investigaciones de Espada sobre las emisoras de Buenos Aires (2018b; 2019). De estos trabajos surge el concepto de "Ecosistema radiofónico Online": En internet conviven distintas plataformas que ofrecen contenidos sonoros que satisfacen las necesidades de entretenimiento e información de las audiencias por las que las radios compiten desde sus inicios. Plataformas musicales que ofrecen música personalizada, podcasters y radios online que aprovechan las reducciones de las barreras de entrada al mercado y producen contenidos de info-entretenimiento, agregadores de streamings y productos radiofónicos, todos se suman a la tradicional oferta de las webs de las emisoras de aire. ¿De qué formas interactúan estos actores en el ecosistema radiofónico web? Las radios tradicionales producen radios online musicales; las radios online producen podcasts; algunos de éstos participan de grillas de programación de radios online; todos comienzan a colgar sus contenidos en plataformas musicales como Spotify o Youtube; y las radios (tradicionales y online) forman parte necesaria de la oferta de agregadores, por nombrar algunas. (Espada, 2017) 
Levatti (2013) trabajó sobre la transformación del rol de la música dentro del medio con la ebullición de plataformas digitales. Entre los estudios sobre el podcasting local se destacan dos trabajos: Porcel y Pereyra (2018) trabajan con el caso cordobés mientras Godinez Galay (2015) con el movimiento de podcasters independientes. Por su parte, Espada (2018c) también trabajó sobre las redes de podcasts surgidas en los últimos años en Buenos Aires.

\section{Conclusiones}

La radio es un objeto definido y trabajado dentro del campo de la comunicación. Existen abordajes variados y alternativos. Desde la perspectiva de los derechos humanos hasta el análisis de su funcionamiento económico; desde las emisoras comunitarias y universitarias hasta la utilización de plataformas y el surgimiento del podcasting. El estudio de la radiofonía como fenómenos social, cultural, tecnológico y económico tiene un gran campo para desarrollarse pero también una larga lista de aportes que ya se han realizado.

Por otro lado, este campo también muestra una riqueza y diversidad geográfica y de género. Tal como puede sostenerse de la radio como medio de comunicación, su capilaridad en la producción de contenidos también se sostiene en la generación de conocimiento sobre sí misma. En su centenario, la radio refuerza su lugar como objeto de estudio, muestra su experiencia pero al mismo tiempo que queda mucho más por decirse y estudiar.

\section{Bibliografía}

- Amdán, F. y Retegui, L. (2017) Estrategias, usos y paradojas en torno a las tic en radios sin fines de lucro: los casos de fm La Tribu y fm Boedo. 
En: Beltrán, R., \& Becerra, M. Sin fines de lucro, con tecnologías y organización. Buenos Aires: UBACyT.

- Amdan, F., Fernández, P. M., Burgos, I. V. G., \& Malcolm, M. S. (2014) Continental y AM 750. Medios y TIC en la Argentina. Estudio sobre adopción de tecnologías de la información en medios de comunicación $/ 1^{a}$ ed./Buenos Aires: Proyecto UBACYT Las tecnologías de los medios de comunicación en el escenario de la convergencia.

- Anselmi, F. y Courau, G. (2014). Rock \& Pop: La imaginación al poder. Buenos Aires, Paidós.

- Atorresi, A. (1995). Los géneros radiofónicos: antología (Vol. 123). Ediciones Colihue SRL.

- Badía, J.A. (2012) En mi vida: autobiografía, Buenos Aires, Planeta.

- Becerra, M., \& Beltrán, R. (2014). Medios y TIC en la Argentina: Estudio sobre adopción de tecnologías de la información en medios de comunicación. Buenos Aires: UBACyT

- Beltrán, R., \& Becerra, M. (2017). Sin fines de lucro, con tecnologías y organización. Buenos Aires: UBACyT.

- Berrade, M. (2009) El Mundo, la radio... un recorrido por el esplendor de la BBC argentina. Corregidor, Buenos Aires.

- Bosetti, O. E. (1994). Radiofonías: palabras y sonidos de largo alcance (Vol. 6). Ediciones Colihue SRL.

- Bosetti, Oscar (1994). Radiofonías. Buenos Aires, Colihue.

- Bosetti, Oscar y Haye, Ricardo (comps) (2015). Las radios universitarias argentinas. Buenos Aires, UNLAM. 
- Bosetti, Oscar y Haye, Ricardo (comps) (2016). Encrucijadas del nuevo milenio: radio, comunicación y nuevas tecnologías. Buenos Aires, UNDAV.

- Bravo, F. (2010) Mi domicilio es el aire. Buenos Aires, Penguin Random House.

- Brunet, M. A. (2003). El eslabón perdido en la historia de la radio. Cuadernos del Sur. Historia, (32), 245-266.

- Casajús, L. (2014). Radios universitarias y redes sociales. Análisis de la gestión de contenidos de la radio universitaria española en las redes sociales (Doctoral dissertation, Universitat Jaume I).

- Casajús, L. (2015). Radios universitarias y redes sociales. Análisis de la gestión de contenidos de la radio universitaria española en las redes sociales (Doctoral dissertation, Universitat Jaume I).

- Casajus, L., \& Guerrero, M. V. (2014). Los jóvenes prosumidores en la radio universitaria 2.0: un perfil en construcción. Edmetic, 3(1), 87-111.

- Casajús, L., \& Martín-Pena, D. (2016). La presencia de las radios universitarias españolas en las redes sociales. Comunicación y Hombre, (12), 291-304.

- Casajus, L., \& Vázquez Guerrero, M. (2014). Los jóvenes prosumidores en la Radio Universitaria 2.0: Un perfil en construcción. EDMETIC, 3(1), 87-111.

- Corda, R., Martínez-Costa, P., \& Müller, M. (2016). Radio, innovación y narrativas transmedias:¿ qué papel juega el sonido en el nuevo ecosistema de los medios multiplataforma. In Ponencia presentada en el Congreso Iberoamericano de Comunicación (AEIC), Madrid. 
- Corda, Rubén, Martínez-Costa, Pilar y Müller, María (2016). "Radio, innovación y narrativas transmedias: ¿qué papel juega el sonido en el nuevo ecosistema de los medios multiplataforma?". Ponencia presentada en el Congreso Iberoamericano de Comunicación (AEIC), Madrid.

- Dougall, A. B., \& Dougall, P. A. (2005). El último broadcaster: la saga de un anglo-criollo en la Argentina (1887-1977). Literature of Latin America.

- Elíades, A. (2003). Historia legal de la radio y la televisión en Argentina. Revista Oficios Terrestres 8 (3). Disponible en: http://perio.unlp.edu.ar/sites/default/files/eliades-_hist_radioytv.pdf

- Espada, A. (2017). Radio en internet y estrategias de negocio: Análisis comparativo de RadioCut, Posta FM, Vorterix Rock y Cienradios. Sphera Publica, 2(17).

- Espada, A. E. (2017). Ecosistema radiofónico online en Argentina. Revista de la Asociación Española de Investigación de la Comunicación, 4(8), 54-62.

- Espada, A. E. (2018). La radio porteña y su estructura de propiedad: análisis de las políticas de comunicación y de la estructura de una industria analógica (1983-2016). Austral Comunicación, 7(1), 121-149.

- Espada, A. E. (2018). Nuevos modelos radiofónicos: las redes de podcast en Argentina. Question.

- Espada, A. E. (2019). Estrategias de negocio para sus webs de las radios más escuchadas de Buenos Aires. adComunica, (17), 67-88.

- Espada, Agustín (2015). Vorterix Rock: hacia la resignificación de los modelos de negocios radiofónicos en Argentina. Revista Comunicación y Medios, 31. Santiago, Universidad de Chile. 
- Fernández, J. (2014). Asedios a la radio. El fin de los medios masivos: El debate continúa, 93-110.

- Fernández, J. L. (1994). Los lenguajes de la radio. Atuel.

- Fernández, J. L. (2003). El hojaldre temporal de lo radiofónico. Figuraciones (185).

- Fernández, J. L. (2008). La construcción de lo radiofónico. Buenos Aires, Argentina: La Crujía.

- Fernández, J. L. (2012). La captura de la audiencia radiofónica. Buenos Aires, Argentina: Liber.

- Fernández, J. L. (2013). Mediatizaciones de sonido en las redes: el Límite Vorterix. Letra. Imagen. Sonido: Ciudad Mediatizada, (10), 150163.

- Fernández, J. L. (2016). O rádio em tempos de mobilidade de networking. Líbero, (34), 65-76.

- Fernández, J. L. (2017). La vida en plataformas: auriculares+ smartphones. Pardo Abril, (2017), 256-273.

- Fernández, J. L. (2018). Plataformas mediáticas: elementos de análisis y diseño de nuevas experiencias. Crujía.

- Fernández, José (1994). Los lenguajes de la radio. Buenos Aires, Paidós.

- Fernández, José (2008). La construcción de lo radiofónico. Buenos Aires, La Crujía.

- Fernández, José (2014). "Asedios a la radio". En: Carlón, Mario y Scolari, Carlos (eds) El fin de los medios masivos. Buenos Aires, La Crujía. 
- Fleitas, Víctor (2015). Historias de aire: hacia una radio que sea fiesta de los sentidos. Entre Ríos, Editorial UNER.

- Gabay, S. P. (2014). Transformaciones de la radio en la era digital: el impacto del nuevo entorno tecnológico (Doctoral dissertation, Universidad Nacional de La Plata).

- Galay, F. G. (2015). Movimiento podcaster: la nueva concreción de la radio libre. Question, 1(46), 135-150.

- Gallo, R. (1991). La radio: ese mundo tan sonoro. Corregidor.

- Gerbaldo, J. (2010). Hacia una cartografía de las radios comunitarias argentinas. Diálogos de la Comunicación, (82), 18.

- Haye, R. (2003). Noticias de un medio cautivante. Otro siglo de radio. La Crujía Ediciones, Buenos Aires.

- Haye, R. (2018). La Radiodifusión Universitaria en la Argentina. Convergencia Revista de Ciencias Sociales, (15).

- Haye, R. M. (2000). Sobre radio y estética. Una mirada desde la filosofía del arte. Convergencia Revista de Ciencias Sociales, (23).

- Haye, R. M. (2003). Otro siglo de radio: noticias de un medio cambiante. La Crujía.

- Horvath, Ricardo (1996). Los avatares de la radio en Argentina. Revista Chasqui, 56, CIESPAL, pp. 56-59.

- Ibarra, D. J. (2017). Transformaciones y persistencias en la comunicación radiofónica: incidencia del uso de Internet y la digitalización en la radio (Doctoral dissertation, Universidad Nacional de la Plata).

- Iglesias, M. (2015). A contramano: modelos de gestión, modos organizativos y estrategias económicas de las emisoras comunitarias 
argentinas en búsqueda de la sustentabilidad (2005-2015) (Doctoral dissertation, Tesis de maestría. Maestría en Industrias Culturales: políticas y gestión, Universidad Nacional de Quilmes (mimeo)).

- Iglesias, M. (2017). Tensiones frente a la sostenibilidad en la Argentina de Cambiemos. RevCom, (5), 34-46.

- Jaimes, D. (2013). La dimensión educativa en las radios comunitarias. Tram [p] as de la Comunicación y la Cultura.

- Jaimes, D. M. (2020). Jóvenes que toman la palabra: radios comunitarias, formación y comunicación popular en la Argentina (20112015).

- Kejval, L. (2017). Investigar desde la comunicación alternativa, popular y comunitaria. Avatares de la comunicación y la cultura, (13).

- Kejval, L. (2017). Reconfiguraciones de la identidad política de las radios comunitarias, populares y alternativas argentinas. RevCom, (5), 6-21.

- Kejval, L. (2018). Una apuesta teórica: comprender las radios comunitarias, populares y alternativas desde el análisis político del discurso/A theoretical proposal: understanding the community, popular and alternative radios from the political discourse analysis. AlgarroboMEL, 7, 1-20.

- Levatti, A. R. (2018) Radio y entretenimiento: la música como rasgo de identidad en el origen de las emisoras AM de Santa $\mathrm{Fe}$ y Paraná. TERRITORIOS de la RADIO SUS HISTORIAS y MEMORIAS, 51.

- Levatti, A. y Lvovich, L (2013) Radio: ¿con la música a otra parte? De la galena a la era digital, Concepción del Uruguay, Universidad Nacional de Entre Ríos - Eduner, 119 pp. 
- Levatti, Ariel y Lvovich, Lea (2013). Radio, ¿con la música a otra parte?: De la galena a la era digital. UNER, Entre Ríos.

- Linares, A. (2017). El despliegue de Radio Nacional de Argentina a internet durante el kirchnerismo. Entre la expansión y la exploración. Viator. Revista científica de comunicación desde los bordes, (4), 81-105.

- Linares, A. (2017). El financiamiento en medios estatales en la Argentina (2003-2009). Questión.

- Linares, A. F. (2020). El desafío del financiamiento de los medios estatales en América Latina. Un debate pendiente y urgente. Revista Internacional de Comunicación y Desarrollo (RICD), 3(12), 33-51.

- Linares, A., \& Mallimaci, M. A. (2019). Los medios estatales de Argentina a partir de la asunción de la alianza Cambiemos en el gobierno nacional (2016-2018). Questión.

- Luque, E. S. M. (2012). Movidito, movidito, juntitos todos juntitos. Apuntes sobre las estrategias expresivas de emisoras orientadas a sectores populares de la ciudad de Córdoba. Question, 1(35), 372-386.

- Marchi, S. (2002). Cinta testigo: la radio por dentro. Sudamericana.

- Martínez Luque, S. (2011). Notas sobre la radio en Córdoba: Continuidades y Transformaciones de la última década. XV Jornadas Nacionales de Investigadores en Comunicación. "Recorridos de Comunicación y Cultura. Repensando prácticas y procesos". Río Cuarto, Córdoba. Mimeo.

- Martínez-Costa, M. P., \& Müller, M. J. (2017). El futuro de la radio musical en el entorno digital en Argentina y España. Revista de la Asociación Española de Investigación de la Comunicación, 4(7), 86-101. 
- Martínez-Costa, M. P., \& Müller, M. J. (2017). El futuro de la radio musical en el entorno digital en Argentina y España. Revista de la Asociación Española de Investigación de la Comunicación, 4(7), 86-101.

- Mata, M. C. (2000). Para una memoria de la radio en Córdoba. Estudios: Centro d Estudios Avanzados, (13), 149-172.

- Mata, María (1993a). "La radio: una relación comunicativa". En Revista Diálogos de la Comunicación, N 35, Lima.

- Mata, María (1993b). “¿Radio popular o comunitaria?”. En Revista Chasqui, N 47, Quito.

- Mata, María (1994). "Hacer radio es producir realidad". En Revista Chasqui, N 49, Quito.

- Mata, María (1998). Saber sobre radio. Revista Signo y Pensamiento, 17 (33), Universidad Javeriana, Colombia, pp. 91-98.

- Mata, María (2000). "Para una memoria de la radio en Córdoba". En Revista Estudios, N 13, Universidad Nacional de Córdoba, Córdoba.

- Mata, María y Scarafía, Silvia (1993). Lo que dicen las radios. Una propuesta para analizar el discurso radiofónico. ALER, Quito.

- Matallana, A. (2006) Locos por la radio : una historia social de la radiofonía en la Argentina, 1923-1947, Buenos Aires, Prometeo.

- Matallana, Andrea (2013). Inventando la radio comercial: Apuntes para una biografía de Jaime Yankelevich. Revista de Instituciones, Ideas y Mercados, 58, pp. 147-166.

- Merkin, M., \& Ulanovsky, C. (1995). Días de radio: historia de la radio argentina. Espasa Calpe. 
- Merkin, Marta, Panno, Juan, Tijman, Gabriela y Ulanovsky, Carlos (1995). Días de radio. Historia de la Radio Argentina. Buenos Aires, Espasa Calpe.

- Milito, C., \& Casajús, L. (2011). Las radios universitarias argentinas en Internet: relevamiento, desarrollos, modelos y enfoques. Question, 1.

- Monje, D. I., Mercadal, S. L., \& Doyle, M. M. (2013). "Otro territorio: emergencia, controversias y perspectiva en torno al emergente sector de medios públicos en Argentina a partir del año 2009". AVATARES de la Comunicación y la Cultura, (6).

- Müller, M. J., Costa, M. P. M., \& Villar, M. (2020). The podcast expansion in public radio: comparative study of Radio Nacional Argentina and Radio Nacional de España (2019). Hipertext. net: Revista Académica sobre Documentación Digital y Comunicación Interactiva, (20), 5.

- Müller, María (2013). "Los programas de máxima audiencia en la radio argentina: características y contenidos de los magacines líderes (2010)”. Tesis, Universidad Austral, Buenos Aires.

- Noguer, J. E. (1985). Radiodifusión en la Argentina (p. 57). Buenos Aires: Editorial Bien Común.

- Olivares, C. (2018) El comienzo de la radiodifusión en San Juan (19301955) 43. TERRITORIOS de Ia RADIO SUS HISTORIAS y MEMORIAS, 37.

- Picco, E. (2016). Apuntes para una historia de los medios de comunicación de Salta. Viator. Revista científica de comunicación desde los bordes, (3), 115-129.

- Porcel, A., \& Quinteros, M. P. (2018). Nuevos medios para viejos fines: Parque Podcast, red de podcasts. COMUNICACION DIGITAL, 58. 
- Portugal, M., \& Yudchak, H. (2008). Hacer radio, Guia integral. Editorial Galerna.

- Radio Rivadavia (1998). 40 Años (1958-1998) de LS5 Radio Rivadavia, Buenos Aires, Rivadavia.

- Rodilla, C. G. A. (2016). La radio y la televisión estatal em Argentina: historia, regulación y procesos de cambio (1920-2015). Revista Brasileira de História da Mídia, 5(1).

- Romano, E. (1993). Voces e imágenes en la ciudad: aproximaciones a nuestra cultura popular urbana (Vol. 1). Ediciones Colihue SRL.

- Rotman, A. (2014). Las radios universitarias, Argentina frente a un nuevo paradigma de la comunicación. Martín Pena, D. y Ortiz Sobrino, MA (comps.) Las radios Universitarias en América y Europa. Madrid: Fragua.

- Segura, M. S., Hidalgo, A. L., Kejval, L., Linares, A., Longo, V., Traversaro, N., \& Vinelli, N. A. (2016). Los medios comunitarios ante las nuevas políticas de comunicación. Actas de Periodismo y Comunicación, 2.

- Segura, M. S., Linares, A., Espada, A., Longo, V., Hidalgo, A. L., Traversaro, N., \& Vinelli, N. (2018). La multiplicación de los medios comunitarios, populares y alternativos en Argentina. Explicaciones, alcances y limitaciones. Revista Internacional de Comunicación y Desarrollo (RICD), 2(9), 88-114.

- Smerling, T. (2012). La concentración de la propiedad de los medios en Rosario: 1997/2007.

- Soledad, S. M., Emilia, V., Verónica, L., Natalia, V., Larisa, K., Laura, H. A., ... \& Natalia, T. (2017). Regresión. Las nuevas políticas para medios comunitarios en Argentina. Logos, 24(1). 
- Tobi, Ximena (2007). La radio en los 60: Redefiniciones a partir de la llegada de la TV. Revista Question, 13 (1), Buenos Aires, UNLP.

- Tobi, Ximena (2008). "El origen de la radio: De la radioafición a la radiodifusión". En: Fernández, José (ed.) La construcción de lo radiofónico. Buenos Aires, La Crujía.

- Tobi, Ximena (2010). La construcción de la empresa radiofónica. Revista Letra, Imagen y Sonido, 3 (5), Buenos Aires, UBA.

- Toledo, D. (2018) LV 13 Radio San Luis: marco organizacional durante su primera década de desarrollo (1942-1952) 43. TERRITORIOS de la RADIO SUS HISTORIAS y MEMORIAS, 37.

- Toledo, D. (2018) Objetivos programáticos de LV 13 Radio San Luis durante su inauguración en 1942: una mirada desde los discursos en medios grá cos47. TERRITORIOS de la RADIO SUS HISTORIAS y MEMORIAS, 57.

- Ulanovsky, C. (2007). Siempre los escucho: retratos de la radio argentina en el siglo XXI. Emece Editores.

- Ulanovsky, C., \& Pelayes, S. (2011). La Radio Nacional. Voces de la historia. 1937-2011. Buenos Aires, RTA.

- Ulanovsky, C., Pelayes, S., Lema, G. y Ronzoni, A. (2014) Radio Belgrano (1983-1989), el aire que la democracia nos legó. Buenos Aires, Colihue.

- Ulanovsky, Carlos (2007). Siempre los escucho. Buenos Aires, Emecé.

- Wildau, P. (2014) Bienvenidos a la radio: un viaje apasionante al corazón de Metro... la radio. Buenos Aires, Dunken. 